\section{Uso de talidomida en sangrado recurrente por angiodisplasias gastrointestinales. Caso clínico}

\author{
JORGE VEGA ${ }^{1,2}$, HELMUTH GOECKE ${ }^{1,2}$, \\ $\mathrm{M}^{\mathrm{a}}$ DE LOS ÁNGELES RODRÍGUEZ ${ }^{3}, \mathrm{M}^{\mathrm{a}}$ TERESA VERGARA ${ }^{2,4}$
}

\section{Thalidomide for recurrent gastrointestinal bleeding caused by angiodysplasia. Report of one case}

Chronic hemodialysis patients may have recurrent bleeding from gastrointestinal angiodysplasia, that often is diffusely located in the digestive tract or in places difficult to reach with traditional endoscopes. Therefore, they cannot be locally treated or removed. We report a 70 years old man on chronic hemodialysis, with severe and persistent anemia due to bleeding from angiodysplasia of the small bowel. Despite administration of high doses of erythropoiesis stimulating agents, intravenous iron, folate, B6 and B12 vitamins, his hemoglobin levels were $<6.5 \mathrm{~g} / \mathrm{dL}$, becoming totally dependent on transfusions of red blood cells (up to 46 units per year). Recurrent bleeding was refractory to conventional management and we decided to use thalidomide at doses of 50-100 mg/day achieving rapid control of gastrointestinal bleeding and significant increase of hemoglobin levels, not requiring further transfusions.

(Rev Med Chile 2011; 139: 909-913).

Key words: Angiodysplasia; Renal dialysis; Thalidomide.
${ }^{1}$ Servicio de Medicina Interna, Sección de Nefrología, Hospital Naval Almirante Nef, Viña del

Mar, Chile.

${ }^{2}$ Departamento de Medicina, Escuela de Medicina, Universidad de Valparaíso, Valparaíso Chile.

${ }^{3}$ Servicio de Medicina Interna, Sección de Hematología, Hospital Naval Almirante Nef, Viña del Mar, Chile

${ }^{4}$ Servicio de Medicina Interna, Sección de Gastroenterología, Hospital Naval Almirante Nef, Viña del Mar, Chile.

Recibido el 18 de enero de 2011, aceptado el 26 de mayo de 2011.

Correspondencia a: Dr. Jorge Vega Stieb 5 Norte 1035, Viña del Mar, Chile. Fono: 56-32-2974237 Fax: 56-32-2970050 E-mail: jvegastieb@gmail. com
E n los pacientes en hemodiálisis crónica (HDC) los sangrados gastrointestinales son frecuentes ${ }^{1-3}$. Si bien la mayoría de las veces son originados por enfermedades habituales hay ocasiones en que el empleo de la panendoscopia, colonoscopia larga con intubación ileal y de técnicas radiológicas no logra precisar su causa, originando una hemorragia digestiva (HD) de origen oscuro ${ }^{3-5}$.

En los últimos años la disponibilidad de la cápsula endoscópica inalámbrica y la enteroscopia con mono o doble balón ha permitido aclarar la causa de $70 \%$ de las HD de origen oscuro, originadas en el intestino delgado y frecuentemente producidas por angiodisplasias (ANG) $)^{4,6}$. Estas lesiones vasculares cuando están al alcance endoscópico o son localizadas, pueden tratarse localmente o resecarse el segmento involucrado ${ }^{3,6,7}$. A menudo las ANG están diseminadas en el tubo digestivo y estas tera- pias no son posibles intentándose diversas terapias farmacológicas con resultados variables ${ }^{3,5-13}$. La Talidomida (TAL), medicamento antiangiogénico actualmente autorizado para la terapia del mieloma múltiple, eritema nodoso secundario a lepra, enfermedad de Behcet, enfermedad de injerto versus huésped en trasplantados de médula ósea, enfermedad de Crohn y estomatitis aftosa en pacientes $\mathrm{HIV}(+)^{14}$ se ha utilizado ocasionalmente en pacientes con ANG y sangrados recurrentes y en hemagioendoteliomas con buenos resultados . Hasta la fecha sólo se han comunicado casos aislados o pequeñas series de $\operatorname{casos}^{4,7-10,14-29}$.

Comunicamos el caso de un enfermo en HDC con ANG difusas del tubo digestivo y sangrado recurrente que se hizo dependiente de transfusiones de glóbulos rojos, y fue tratado exitosamente con TAL deteniéndose los sangrados, sin requerir más transfusiones. 


\section{Caso clínico}

Paciente de 68 años que el año 2004 sufrió una gangrena de Fournier y requirió de medicina hiperbárica y colostomía transitoria. En abril de 2006 ingresó a hemodiálisis (HDC) por nefropatía diabética. Tenía antecedentes de diabetes mellitus, hipertensión arterial, litiasis urinaria, úlcera pépti$\mathrm{ca}$, ateroesclerosis coronaria y periférica (puentes aorto coronario/aorto bifemoral el 2002), daño hepático crónico y embolia pulmonar. El año 2006, fue hospitalizado 2 veces por HD alta encontrándose una úlcera duodenal activa, que se trató con inyección local de adrenalina más omeprazol y en la otra ocasión, ANG en bulbo duodenal que se coagularon. Recibió 10 unidades de glóbulos rojos (UGR).

$\mathrm{Al}$ año siguiente (2007) fue hospitalizado 2 veces por HD baja sin que se encontraran lesiones en la endoscopia alta y colonoscopia. La video-cámara mostró angiodisplasias en intestino delgado medio $\mathrm{y}$ en duodeno. Estas últimas fueron tratadas con argón-plasma. El paciente se mantuvo persistentemente anémico (hemoglobina promedio $7,7 \mathrm{~g} / \mathrm{dL}$ en los exámenes mensuales de rutina) a pesar del uso de eritropoyetina (EPO) 12.000 unidades/ semanales, hierro ev $400 \mathrm{mg} / \mathrm{mensuales,} \mathrm{ácido} \mathrm{fólico}$ y vitaminas B. Ese año recibió $25 \mathrm{UGR}$ (promedio 2 mensuales).

El año 2008 fue hospitalizado en 2 ocasiones por HD y mantuvo sangrado digestivo persistente. A pesar de ser transfundidas 45 UGR (promedio 3,8 mensuales) y administrado EPO (12.00024.000 unidades semanales), hierro, vitaminas B6 y B12 y folatos, la hemoglobina promedio fue $6,4 \mathrm{~g} / \mathrm{dL}$ y la saturación de transferrina (SAT) no superó el $26 \%$. A raíz de un incremento progresivo en el volumen corpuscular medio con niveles de folatos y B12 elevados, se efectuó un mielograma que evidenció signos de mielodisplasia. Un scanner mostró un bazo de $14,5 \mathrm{~cm}$ de longitud que hizo sospechar hiperesplenismo.

El año 2009 continuó con sangrado bajo frecuente y la anemia empeoró con hemoglobinas promedio de $6,3 \mathrm{~g} / \mathrm{dL}$ a pesar de recibir 12.000 U semanales de EPO (posteriormente 200 ug de metoxipolietilenglicol-epoetina beta) y 46 unidades de glóbulos rojos (promedio 3,8 mensuales; rango: $1-14$ ). La SAT se mantuvo siempre $<17 \%$.

El año 2010, la anemia se acentuó a pesar de incrementar la dosis del activador del receptor de
EPO a $400 \mu \mathrm{g} / \mathrm{mensuales,} \mathrm{requiriendo} 15$ UGR en los 2 primeros meses sin superar un hematocrito de $19 \%$. Se decidió ensayar el empleo de TAL por sus efectos antiangiogénicos, con la autorización del paciente, indicando $1 / 2$ tableta diaria $(50 \mathrm{mg}$ ) el 25 de febrero de 2010. En marzo el paciente no requirió de transfusiones, dejó de sangrar externamente y el hematocrito subió a 21,6\%. El 29 de marzo, se trombosó la fístula arteriovenosa que usaba para dializarse lo que se atribuyó a los efectos trombogénicos de TAL, suspendiéndose la droga. En los días siguientes reapareció el sangrado digestivo, cayó el hematocrito a $16 \%$ requiriendo de 6 UGR. Se reinstaló la TAL 100 mg/día el 20 de abril. En los 2 meses siguientes no requirió de transfusiones ni sangró externamente y la hemoglobina subió a 10,6 g/dL. Posteriormente, falleció como consecuencias de un traumatismo encefalocraneano por caída en el baño.

\section{Discusión}

Las ANG gastrointestinales son malformaciones vasculares que pueden ser hereditarias o adquiridas. Estas pueden localizarse en estómago (ectasias vasculares antrales gástricas) dándole a la mucosa un aspecto de sandía o en intestino. Los sitios más frecuentemente afectados son colon e íleon terminal. En este paciente se las encontró en duodeno e intestino medio. La patogénesis de las ANG no está clara. Las ANG están cubiertas por endotelio y tienen escaso músculo liso por lo que son muy frágiles. Las ANG adquiridas se asocian más frecuentemente a Enfermedad de von Willebrand, cardiopatías valvulares (estenosis aórtica) e insuficiencia renal crónica (IRC) avanzada, como en este paciente. En sujetos con IRC las ANG pueden manifestarse como anemia crónica resistente a la EPO, anemia ferropriva a pesar del aporte de hierro endovenoso con sangre oculta en deposiciones, hallazgo durante una endoscopia y sangrado externo agudo o recurrente con dependencia de transfusiones ${ }^{3,5}$. En este paciente se presentó de todas estas formas. Su prevalencia en los pacientes con IRC no se conoce. En un estudio en que se efectuó panendoscopia en 260 pacientes previo a un trasplante renal se encontró ANG en $4,4 \%{ }^{30}$. En otro estudio en 727 pacientes con HDA se encontró que las ANG eran la causa en 1,3\% del total de pacientes y en $13 \%$ de quienes sufrían $I C^{31}$. Las ANG son la etiología de 30\% de las HD 
altas, 20\% de los sangrados bajos y la etiología más frecuente de las HD bajas recurrentes en pacientes en HDC. En el 70\% de los enfermos las lesiones son múltiples. El sangrado es autolimitado en 90\% de los casos pero puede amenazar la vida. Las ANG son una patología propia de sujetos mayores de 60 años y se han observado tanto en pacientes en etapa pre-dialítica como en HDC o diálisis peritoneal.

Su diagnóstico puede hacerse mediante panendoscopia, colonoscopia, cápsula endoscópica inalámbrica, enema bajo scanner, observación durante la cirugía, enteroscopia de doble balón o intraoperatoria, angiografía o en una pieza resecada ${ }^{13}$.

Las opciones terapéuticas son: terapia endoscópica (fotoablación con laser, coagulación con argón-plasma, electrocoagulación bipolar o multipolar), cirugía y drogas ${ }^{3,9,11}$. Las ANG pueden ser recalcitrantes a la terapia endoscópica, estar diseminadas por el tubo digestivo o localizarse en áreas difíciles de acceder con endoscopia convencional como sucedió en este enfermo. Los pacientes pueden presentar comorbilidades que aumenten el riesgo de los procedimientos endoscópicos y quirúrgicos. En este paciente se desechó practicar una endoscopia con doble balón por los riesgos de la anestesia general. En enfermos semejantes se han ensayado diversas drogas con resultados variables, a menudo frustrantes, como estrógenos, progesterona, vasopresina, concentrado de factor VIII, factor VII recombinante, desmopresina, danazol, ácido épsilon-amino-caproico y tranexámico, betabloqueadores y análogos de la somatostatina (Octreotide ${ }^{6,8,13}$. Con este último fármaco se han obtenido mejores resultados, disminuyendo el número de transfusiones y las hospitalizaciones, pero con el inconveniente de requerir administrarse por vía subcutánea cada 8 ó 12 horas, aunque existe una presentación de depósito por vía intramuscular mensual de alto costo ${ }^{5-7,11}$.

Recientemente se han comunicado experiencias exitosas con TAL, droga con efectos antiangiogénicos y antiinflamatorios ${ }^{4,7,10,14-19,23,26}$. Su uso ha permitido detener los sangrados en corto tiempo, disminuyendo o eliminando las transfusiones y las hospitalizaciones ${ }^{10,14,16-18,20}$. Su efecto beneficioso no sólo se logra mientras se administra la droga sino que puede persistir por meses o años desde su suspensión, aunque los sangrados pueden reaparecer obligando a reinstaurarla ${ }^{16,22}$. Las dosis han variado entre $50-400 \mathrm{mg} /$ día consiguiéndose beneficios con $100 \mathrm{mg} /$ día. Se recomienda iniciar el tratamiento con 50/100 mg/día como en este paciente ${ }^{32}$. La duración de su administración ha sido variable, comunicándose terapias de hasta 12 meses $^{2,15,17,20}$. Las principales limitaciones que han obligado a suspender la droga son la fatiga, neuropatía periférica, constipación y rash cutáneo ${ }^{15}$. Se ha recomendado utilizar la TAL en la noche para controlar la fatiga ${ }^{2}$. También se han comunicado eventos tromboembólicos en pacientes que están con anticoagulación o antiplaquetarios, insuficiencia hepática aguda e hiperkalemia fatal en pacientes en HDC que han usado TAL para el prurito urémico ${ }^{12,21,32}$. A este frágil paciente, que se dializaba en la unidad de diálisis del hospital, se lo vigiló estrechamente por la eventualidad de la aparición de algún efecto secundario, cosa que no ocurrió (salvo la oclusión de su acceso vascular para hemodiálisis) y se midió sus parámetros hematológicos semanalmente. En IRC no es necesario reducir las dosis de TAL. El empleo de la cápsula endoscópica como monitoreo del tratamiento con TAL ha permitido comprobar una reducción del número y tamaño de las $\mathrm{ANG}^{15,18,26}$. La lenalidomida, actualmente en uso en pacientes con mieloma múltiple es un derivado de TAL de mayor poder antiangiogénico y menores efectos secundarios, que probablemente se utilice en el futuro en sangrados por ANG (hay un caso comunicado con buenos resultados) $)^{25}$.

En un estudio experimental se demostró que la TAL suprime la expresión del factor de crecimiento vascular endotelial (VEGF) en las células endoteliales inhibiendo la formación de angiodisplasias ${ }^{33}$. Las ANG son lesiones que expresan VEGF, receptor 1 del VEGF y el factor básico de crecimiento fibroblástico ${ }^{2,14}$. El VEGF es un péptido angiogénico secretado en respuesta a la hipoxia que estimula la proliferación de las células endoteliales. Los pacientes con ANG se caracterizan por tener niveles elevados de $\mathrm{VEGF}^{7}$. Estos niveles se reducen durante el tratamiento con $\mathrm{TAL}^{2}$. Se piensa que al reducir los niveles de VEGF se reduce la formación de ANG y disminuye el número y volumen de las existentes reduciéndose el riesgo de sangrado ${ }^{2}$.

\section{Conclusiones}

Este paciente en HDC presentó una anemia originada en el sangrado crónico de ANG intesti- 
nales, agravada por la presencia de hiperesplenismo, mielodisplasia, daño hepático y renal crónico. La terapia con TAL permitió prescindir de las transfusiones, detener el sangrado e incrementar significativamente la hemoglobina. Comunicamos esta experiencia, porque dada la alta prevalencia de pacientes añosos en HDC (susceptibles a tener angiodisplasias) esta situación podría no ser tan infrecuente y el uso de esta droga podría ser de gran utilidad como lo fue en este paciente.

\section{Referencias}

1. Karagiannis S, Goulas S, Kosmadakis G, Galanis P, Arvanitis D, Boletis J, et al. Wireless capsule endoscopy in the investigation of patients with chronic renal failure and obscure gastrointestinal bleeding (preliminary data). World J Gastroenterol 2006; 12: 5182-5.

2. Korsets A, Gafter U, Chagnac A, Zingerman B, Morduchovitz V, Ori Yaacov. Thalidomide: a treatment option for bleeding GI angiodisplasias in dialysed patients. NDT Plus 2008; 5: 354-6.

3. Fabián G, Szigeti Nóra, Kovács T, Nagy Judit. An unusual multiplex cause of severe gastrointestinal bleeding in a haemodialysed patient. Nephrol Dial Transplant 2000; 15: 1869-71.

4. Bélard E, Foss CH, Christensen LA, Schmidt P, Nojgaard C. Thalidomide therapy for gastrointestinal angiodysplasia. Ugeskr Laeger 2009; 171: 3454-5.

5. Molina Infante J, Pérez Gallardo B, Hernández Alonso M, Mateos Rodríguez JM, Dueñas Sadornil C, Fernández Bermejo M. Octreotide long acting release for severe obscure gastrointestinal haemorrhage in elderly patients with serious comorbidities. Med Clin (Barc) 2009; 133: 667-70.

6. Molina Infante J, Pérez Gallardo B, Fernández Bermejo M. Update on medical therapy for oscure gastrointestinal hemorrhage. Rev Esp Enferm Dig 2007; 99: 457-62.

7. Almadi M, Ghali PM, Constantin A, Galipeau J, Szilagyi. Recurrent obscure gastrointestinal bleeding: dilemmas and success with pharmacological therapies. Case series and review. Can J Gastroenterol 2009; 23: 625-31.

8. Selinger CP, Ang YS. Gastric antral vascular ectasia (GAVE): un update on clinical presentation, pathophysiology and treatment. Digestion 2008; 77: 131-7.

9. Bauditz J, Lochs H. Angiogenesis and vascular malformations: antiangiogenic drugs for treatment of gastrointestinal bleeding. World J Gastroenterol 2007; 13: 5979-84.

10. Hirri HM, Green PJ, Lindsay J. Von Willebrand's disease and angiodysplasia treated with thalidomide. Haemophilia 2006; 12: 285-6.

11. Brown C, Subramanian V, Wilcox CM, Peter S. Somatostatin analogues in the treatment of recurrent bleeding from gastrointestinal vascular malformations: an overview and systematic review of prospective observational studies. Dig Dis Sci 2010; 55: 2129-34.

12. Molina-Infante J, Pérez-Gallardo B. Somatostatin analogues for bleeeding gastrointestinal angiodisplasias: when should thalidomide be prescribed? Dig Dis Sci 2011; 56: 266-7.

13. Szilagyi A, Ghali MP. Pharmacological therapy of vascular malformations of the gastrointestinal tract. Can $\mathrm{j}$ Gastroenterol 2006; 20: 171-8. A14. A27.

14. Shurafa M, Kamboj G. Thalidomide for the treatment of bleeding angiodisplasias. Am J Gastroenterol 2003; 98: 2212.

15. Kamalapom P, Saravanan R, Cirocco M, May G, Kortan P, Kandel G, et al. Thalidomide for the treatment of chronic gastrointestinal bleeding from angiodysplasias: a case series. Eur J Gastroenterol Hepatol 2009; 21: 1347 50.

16. Dabak V, Kuriakose P, Kamboj G, Shurafa M. A pilot study of thalidomide in recurrent GI bleeding due to angiodisplasias. Dig Dis Sci 2008; 53: 1632-5.

17. Heidt J, Langers AM, van der Meer FJ, Brouwer RE. Thalidomide as treatment for digestive tract angiodisplasias. Neth J Med 2006; 64: 425-8.

18. Bauditz J, Lochs H, Voderholzer W. Macroscopic appearance of intestinal angiodisplasias under antiangiogenic treatment with thalidomide. Endoscopy 2006; 38: 10369.

19. de Koning DB, Drenth JP, Friederich P, Nagengast FM. Thalidomide for the treatment of recurrent gastrointestinal blood loss due to intestinal angiodisplasias. Ned Tijdschr Geneeskd 2006; 150: 1994-7.

20. Dunne KA, Hill J, Dillon JF. Treatment of chronic transfusion-dependent gastric antral vascular ectasia (watermelon stomach) with thalidomide. Eur J Gastroenterol Hepatol 2006; 18: 455-6.

21. Mimidis K, Kaliontzidou M, Tzimas T, Papadopoulos V. Thalidomide for treatment of bleeding angiodisplasias during hemodialysis. Renal Failure 2008; 30: 1040-1.

22. Serralta de Colsa D, Arjona Medina I, García Marín A, Sanz Sánchez M, Turégano Fuentes F. Invalidating and recurrent gastrointestinal bleeding caused by intestinal angiodysplasia: treatment with thalidomide. Rev Esp Enferm Dig 2008; 100: 306-7.

23. Fernández Salazar LI, Velayos Jiménez B, Fernández Galante I, Aller de la Fuente R, González Hernández JM. Efficacy of thalidomide in digestive bleeding caused by 
angiodisplasias. Rev Esp Enferm Dig 2009; 101: 368-73.

24. Kirkham SE, Lindley KJ, Elawad MA, Blanshard C, Shah $\mathrm{N}$. Treatment of multiple small angiodisplasias causing severe life-theattening bleeding with thalidomide. J Pediatr Gastroenterol Nuth 2006; 42: 585-7.

25. Bowcock SJ, Patrick HE. Lenalidomide to control gastrointestinal bleeding in hereditary haemorrhagic telangiectasia: potential implications for angiodisplasias? Br J Haematol 2009; 146: 2202.

26. Jiménez-Saenz M, Romero-Vázquez J, Caunedo-Álvarez A, Maldonado-Pérez B, Gutiérrez JM. Beneficial effects and reversión of vascular lesions by thalidomide in a patient with bleeding portal hypertensive enteropathy. Dig Liver Dis 2010; 42: 232-3.

27. Raphael C, Hudson E, William L, Lester JF, Savage PM. Successful treatment of metastatic hepatic epithelioid hemangioendothelioma with thalidomide: a case report. J Med Case Reports 2010; 4: 413.

28. Mascarenhas RC, Sanghvi AN, Friedhander L, Geyer SJ,
Beasley HS, Van Thiel DH. Thalidomide inhibits the grow and progression of hepatic epithelioid hemangioendothelioma. Oncology 2004; 67: 471-5.

29. Salech F, Valderrama S, Nervi B, Rodríguez JC, Oksenberg D, Kock A, et al. Ann Hepatol 2011; 10: 99-102.

30. Sotoudehmanesh R, Ali Asgari A, Ansari R, Nouraie M. Endoscopic findings in end-stage renal disease. Endoscopy 2003; 36: 502-5.

31. Chalasani N, Cotsonis G, Wilcox CM. Upper gastrointestinal bleeding in patients with chronic renal failure: role of vascular ectasia. Am J Gastroenterol 1996; 91: 2329-32.

32. Izzedine H, Launay-Vacher V, Deray G. Thalidomide for the nephrologis. Nephrol Dial Transplant 2005; 20: 2011-2.

33. Chen HM, Ge ZZ, Liu WZ, Lu H, Xu CH, Fang JY, et al. The mechanisms of thalidomide in treatment of angiodysplasia due to hypoxia. Zhonghua Nei Ke Za Zhi 2009; 48: 295-8. 